\title{
Actinobacillus pleuropneumoniae RTX-toxins: uniform designation of haemolysins, cytolysins, pleurotoxin and their genes
}

\author{
J. Frey, ${ }^{1 *}$ J. T. Bosse, ${ }^{2}$ Y.-F. Chang, ${ }^{3}$ J. M. Cullen, ${ }^{4}$ B. Fenwick,,${ }^{5}$ G. F. Gerlach,${ }^{16}$ \\ D. Gygi,${ }^{6}$ F. Haesebrouck, ${ }^{7}$ T. J. InZana,${ }^{8}$ R. Jansen,,${ }^{9}$ E. M. Kamp, ${ }^{9}$ J. Macdonald,${ }^{4}$ \\ J. I. MacInnes, ${ }^{2}$ K. R. Mittal, ${ }^{10}$ J. Nicolet, ${ }^{1}$ A. N. RyCroft,${ }^{11}$ R. P. A. M. Segers, ${ }^{12}$ \\ M. A. Smits, ${ }^{9}$ E. STendaeK, ${ }^{13}$ D. K. StrucK, ${ }^{14}$ J. F. van den BosCh, ${ }^{12}$ P. J. Willson ${ }^{15}$ \\ and R. YounG ${ }^{14}$
}

${ }^{1}$ Institute for Veterinary Bacteriology, University of Berne, Länggass-Strasse 122, CH-3012 Berne, Switzerland

${ }^{2}$ University of Guelph, Guelph, Ontario, Canada

${ }^{3}$ College of Veterinary Medicine, Cornell University, Ithaca, NY, USA

${ }^{4}$ Department of Veterinary Pathology, University of Glasgow, Glasgow, UK

${ }^{5}$ Department of Pathology and Microbiology, Kansas State University, Manhattan, KS, USA

${ }^{6}$ Department of Pathology, Cambridge University, Cambridge, UK

${ }^{7}$ Faculty of Veterinary Medicine, University of Gent, Gent, Belgium

${ }^{8}$ Virginia Polytechnic, Blacksburg, VA, USA

${ }^{9}$ Central Veterinary Institute, Lelystad, The Netherlands

${ }^{10}$ Faculté de médécine vétérinaire, Université de Montréal, St-Hyacinthe, Québec, Canada

${ }^{11}$ Royal Veterinary College, UCL, London, UK

${ }^{12}$ Intervet International $B V, A A$ Boxmeer, The Netherlands

${ }^{13}$ Statens Veterinaere Serumlaboratorium, Kobenhavn, Denmark

${ }^{14}$ Texas A\&M University, College Station, TX, USA

${ }^{15}$ Veterinary Infectious Disease Organization, University of Saskatchewan, Saskatoon, Saskatchewan, Canada

${ }^{16}$ Institute for Microbiology and Infectious Diseases, Veterinary School, Hanover, Germany

(Received 13 April 1993; accepted 23 April 1993)

The three different pore-forming RTX-toxins of Actinobacillus pleuropneumoniae are reviewed, and new and
uniform designations for these toxins and their genes are proposed. The designation ApxI (for Actinobacillus
pleuropneumoniae RTX-toxin I) is proposed for the RTX-toxin produced by the reference strains for serotypes 1 ,
$5 \mathrm{a}, 5 \mathrm{~b}, 9,10$ and 11 , which was previously named haemolysin I (HlyI) or cytolysin I (ClyI). This protein is strongly
haemolytic and shows strong cytotoxic activity towards pig alveolar macrophages and neutrophils; it has an
apparent molecular mass in the range 105 to $110 \mathrm{kDa}$. The genes of the apxI operon will have the designations
apxIC, apxIA, apxIB, and apxID for the activator, the structural gene and the two secretion genes respectively.
The designation ApxII is proposed for the RTX-toxin which is produced by all serotype reference strains except
serotype 10 and which was previously named App, HyII, ClyII or Cyt. This protein is weakly haemolytic and
moderately cytotoxic and has an apparent molecular mass between 103 and $105 \mathrm{kDa}$. The genes of the apxII
operon will have the designations apxIIC for the activator gene and apxIIA for the structural toxin gene. In the
apxII operon, no genes for secretion proteins have been found. Secretion of ApxII seems to occur via the products
of the secretion genes apxIB and apxID of the apxI operon. The designation ApxIII is proposed for the non-
haemolytic RTX-toxin of the reference strains for serotypes $2,3,4,6$ and 8 , which was previously named
cytolysin III (ClyIII), pleurotoxin (Ptx), or macrophage toxin (Mat). This protein is strongly cytotoxic and has
an apparent molecular mass of $120 \mathrm{kDa}$. The genes of the apxIII operon have the designations apxIIIC, apxIIIA,
apxIIIB and apxIIID for the activator gene, the structural gene and the two secretion genes respectively.

*Author for correspondence. Tel. +41 31631 2484; fax +41 31631 2634; e-mail JFREY@VBI.UNIBE.CH. 


\section{Introduction}

Actinobacillus pleuropneumoniae is a Gram-negative bacterium belonging to the family Pasteurellaceae. It is the aetiological agent of swine pleuropneumonia, a severe disease causing large economic losses in industrialized swine production (Shope, 1964; Nicolet, 1992). Among the twelve different serotypes, three different exotoxins which belong to the group of poreforming RTX-toxins (Welch, 1991) have been detected (Frey \& Nicolet, 1988a, 1990; Frey et al., 1991 b, 1992; Chang et al., 1989; Rycroft et al., 1991a; Kamp et al., 1991; Smits et al., 1991; Lalonde et al., 1989; Jansen et $a l ., 1993,1992 b$; Macdonald \& Rycroft, 1992; van den Bosch, 1991; Gygi et al., 1990). Two of these RTX-toxins possess both haemolytic and cytotoxic activity (Frey \& Nicolet, 1988 a; Rosendal et al., 1988; Kamp et al., 1991) while the third has cytotoxic, but not haemolytic activity (Rycroft et al., 1991a; Kamp et al., 1991). The RTXtoxins have molecular masses of $100-120 \mathrm{kDa}$ and can be distinguished serologically by polyclonal and monoclonal antibodies (Frey et al., 1992; Kamp et al., 1991; Rycroft et al., 1991 a; Kamp \& van Leengoed, 1989). Reference and field strains of several serotypes produce two different RTX-toxins (Frey et al., 1992; Kamp et al., 1991; Rycroft et al., $1991 a$; Jansen et al., 1992a,b, 1993), a situation which is rather unusual among RTXtoxin-producing bacteria and which created confusions in the serological distinction of different RTX-proteins. All RTX-toxins of A. pleuropneumoniae have been shown to be major immunogenic factors in experimentally and naturally infected pigs (Devenish et al., $1990 a, b$; Ma \& Inzana, 1990; Frey \& Nicolet, 1991; Rycroft et al., $1991 a$ ) and seem to be important virulence factors (Inzana et al., 1991; Kamp et al., 1991; Stine et al., 1991; Rycroft et al., 1991 a, b; Udeze \& Kadis, 1992; Devenish et al., 1992; Dom et al., 1992). They have also been shown to be involved in the induction of protective immunity and are suggested to be important components in vaccines against swine pleuropneumonia (Bosse $e t$ al., 1992; Bhatia et al., 1991; Inzana et al., 1991; Rycroft et al., 1991 a; Rossi Campos et al., 1992; van den Bosch et al., 1992; van den Bosch, 1991; Devenish et al., 1990a; Fedorka Cray et al., 1990).

In general, the operons encoding RTX-toxins consist of four contiguous genes in the order C A B D. The A gene encodes the structural toxin protein, the $\mathrm{C}$ gene encodes an activator protein which is involved in fatty acylation in the conversion of the protoxin to active toxin, and the B and D genes encode membraneassociated proteins for secretion of the toxins (Issartel $e t$ al., 1991; Welch, 1991; Felmlee et al., 1985). While this is also true for several RTX-operons of $A$. pleuropneumoniae (Gygi et al., 1992; Jansen et al., 1993, $1992 b$ ), partial RTX-operons containing two genes have been found in $A$. pleuropneumoniae and Actinobacillus suis (Burrows \& Lo, 1992; Frey et al., 1993; Chang et al., 1991; Jansen et al., $1992 a, b$; Smits et al., 1991). The presence of different combinations of the three RTXtoxins in strains from the same species originally led to considerable confusion, probably because of the very similar molecular masses of these toxins. Due to the

Table 1. New designations and synonyms used for the various $R T X$-toxins found in A. pleuropneumoniae

\begin{tabular}{|c|c|c|c|c|c|c|c|c|c|}
\hline $\begin{array}{l}\text { Name of } \\
\text { toxin }\end{array}$ & $\begin{array}{l}\text { Protein/ } \\
\text { gene } \\
\text { symbols }\end{array}$ & $\begin{array}{l}\text { Name of } \\
\text { toxin }\end{array}$ & $\begin{array}{l}\text { Protein/ } \\
\text { gene } \\
\text { symbols }\end{array}$ & $\begin{array}{l}\text { Name of } \\
\text { toxin }\end{array}$ & $\begin{array}{l}\text { Protein/ } \\
\text { gene } \\
\text { FCRsymbols }\end{array}$ & $\begin{array}{l}\text { Name of } \\
\text { toxin }\end{array}$ & $\begin{array}{l}\text { Protein/ } \\
\text { gene } \\
\text { symbols }\end{array}$ & $\begin{array}{l}\text { Name of } \\
\text { toxin }\end{array}$ & $\begin{array}{l}\text { Protein/ } \\
\text { gene } \\
\text { symbols }\end{array}$ \\
\hline RTX-toxin I & $\begin{array}{l}\text { ApxI } \\
\text { apxIC } \\
a p x I A \\
\text { apxIB } \\
\text { apxID }\end{array}$ & Haemolysin I & $\begin{array}{l}\text { Hlyl }^{e} \\
h_{l y I C^{f g}} \\
h_{l y I A^{f, g}} \\
\text { hlyIB }^{g} \\
h l y I D^{g}\end{array}$ & Cytolysin I & $\begin{array}{l}\text { ClyI }^{k} \\
\text { clyIC } \\
\text { clyIA } \\
\operatorname{clyIB}^{i} \\
\operatorname{clyID}^{i}\end{array}$ & & $\begin{array}{l}a p p B^{d} \\
a p p D^{d}\end{array}$ & & \\
\hline RTX-toxin II & $\begin{array}{l}\text { ApxII } \\
\text { apxIIC } \\
a p x I I A\end{array}$ & Haemolysin II & $\begin{array}{l}\text { HlyII }^{e} \\
h l y I I C^{f} \\
h l y I I A^{f}\end{array}$ & Cytolysin II & $\begin{array}{l}\text { ClyII }^{k} \\
\text { clyIIC } \\
\text { clyIIA }\end{array}$ & Haemolysin & $\begin{array}{l}\text { App } \\
\operatorname{app} C^{c} \\
a p p A^{c}\end{array}$ & Cytolysin & $\begin{array}{l}\mathrm{Cyt}^{a} \\
\operatorname{cyt} C^{a} \\
\operatorname{cyt} A^{a}\end{array}$ \\
\hline RTX-toxin III & $\begin{array}{l}\text { ApxIII } \\
\text { apxIIIC } \\
\text { apxIIIA } \\
\text { apxIIIB } \\
\text { apxIIID }\end{array}$ & Pleurotoxin & $\begin{array}{l}\operatorname{Ptx}^{m} \\
p t x A^{l}\end{array}$ & Cytolysin III & $\begin{array}{l}\text { ClyIII }^{k} \\
\text { clyIIIC } \\
\text { clyIII } \\
\operatorname{clyIIIB}^{h j} \\
\operatorname{clyIIID}^{h}\end{array}$ & & & $\begin{array}{l}\text { Macrophage- } \\
\text { toxin }\end{array}$ & $\mathrm{Mat}^{b}$ \\
\hline
\end{tabular}

* References: a, Anderson et al. (1991); b, van den Bosch et al. (1992); c, Chang et al. (1989); d, Chang et al. (1991); e, Frey \& Nicolet (1988a); $f$, Frey et al. (1992); $g$, Gygi et al. (1992); $\boldsymbol{h}$, Jansen et al. (1992a); $\boldsymbol{i}$, Jansen et al. (1992b); $\boldsymbol{j}$, Jansen et al. (1993); $\boldsymbol{k}$, Kamp et al. (1991); $l$, Macdonald \& Rycroft (1992); $\boldsymbol{m}$, Rycroft et al. (1991a). 
intense research efforts on $A$. pleuropneumoniae RTX toxins which have been made simultaneously by various groups during the last few years, several different toxin names and gene designations have been used. A combination of the results from the molecular characterization at the genetic level and serological and toxicity studies has resulted in the present understanding of RTX-toxins and genes in A. pleuropneumoniae strains. The researchers working on $A$. pleuropneumoniae RTX toxins therefore made the effort to agree upon a common, uniform nomenclature for these toxins which was proposed at the Fallen Leaf Lake Conference 1992 on 'Bacterial Virulence Mechanisms'. These new designations and their synonyms are listed in Table 1 and are briefly described below. They are abbreviated by Apx, standing for Actinobacillus pleuropneumoniae RTXtoxins. ApxI, ApxII and ApxIII will be used according to numbering used in previously published work (Jansen $e t$ al., 1992a; Smits et al., 1991; Kamp et al., 1991; Frey \& Nicolet, 1988a, 1990). For RTX toxins from other species the same strategy for abbreviations could be used.

\section{ApxI (previously HlyI or ClyI)}

A. pleuropneumoniae-RTX-toxin I (ApxI) was initially described as the strongly haemolytic haemolysin I (HlyI), purified from serotype 1 type strain 4074 as an active protein with an apparent molecular mass of $105 \mathrm{kDa}$ (Frey \& Nicolet, 1988 a,b). ApxI was also found to be cytotoxic for alveolar macrophages and neutrophils and therefore it was also named cytolysin I (ClyI) (Kamp et al., 1991). ApxI was found to be present in the reference strains of serotypes 1, 5a, 5b, 9, 10 and 11 (Frey \& Nicolet, 1990; Frey et al., 1992; Kamp et al., 1991). In these reference strains the operon of ApxI contains four genes: apxIC, the activator gene; apxIA, the structural toxin gene; and $a p x I B$ and $a p x I D$, the secretion genes (Table 2). These genes were previously named hlyIC, $h l y I A, h l y I B$ and $h l y I D$ respectively in serotype 1 (Gygi et al., 1990, 1992; Frey et al., 1993) or clyIC, clyIA, clyIB and clyID in serotype 9 (Jansen $e t$ al., $1992 a, b$; Smits $e t$ $a l ., 1991)$. The apxIA genes of serotypes 1,9 and 11 are similar whereas they show allelic variation in serotypes 5a, 5b and 10 (Jansen et al., 1992b; Frey et al., 1993). The genes $a p x I B$ and $a p x I D$ correspond to the genes $a p p B$ and $a p p D$, which were initially believed to be the secretion genes of ApxII (initially named App) (Chang et al., 1991). The apxI operon is transcribed into two RNA species: a major one of $3.5 \mathrm{~kb}$ which is $\mathrm{Ca}^{2+}$ inducible and contains apxIC and apxIA, and a minor transcript of $7.5 \mathrm{~kb}$ which is not induced by $\mathrm{Ca}^{2+}$ and contains the whole operon apxICABD (Gygi et al., 1992). The reference strains of serotypes $2,4,6,7,8$ and
12 do not possess apxIC and apxIA and therefore these serotypes do not produce ApxI. Instead these serotypes possess a truncated apxI operon containing $a p x I B$ and apxID and the C-terminal end of apxIA (Frey et al., 1993; Jansen et al., 1992b). The serotype 3 reference strain lacks the entire apxI operon (Frey et al., 1993; Jansen et al., 1992b). The structural gene apxIA has been sequenced and was shown to be similar to the Escherichia coli haemolysin gene $h l y A$ and to a lesser extent to Pasteurella haemolytica leukotoxin gene lktA (Frey et al., $1991 b$, 1993). apxIA encodes the protein ApxIA, which has a molecular mass of $110.2 \mathrm{kDa}$ as deduced from the DNA sequence. It contains 13 glycine-rich repeats and binds ${ }^{45} \mathrm{Ca}^{2+}$ (Frey et al., 1991 b). This binding is required for haemolytic activity and binding to neutrophils (van Leengoed \& Dickerson, 1992; Devenish \& Rosendal, 1991).

\section{ApxII (previously HlyI, ClyII, App or Cyt)}

ApxII was initially described as the weakly haemolytic toxin HlyII (haemolysin II) with an apparent molecular mass of $105 \mathrm{kDa}$ isolated from serotype 2 reference strain S1536 (Frey \& Nicolet, 1988a, 1990; Frey et al., 1991a). ApxII was identified as a $103 \mathrm{kDa}$ protein by monoclonal antibodies in the reference strains of all serotypes except 10 (Kamp et al., 1991). Besides its haemolytic activity, ApxII was also found to be cytotoxic for alveolar macrophages and neutrophils and was designated cytolysin II (ClyII) (Kamp et al., 1991). The genes encoding the activator and the structural protein of ApxII were first cloned from serotype 5 and sequenced (Chang et al., 1989) and designated $a p p C$ and appA respectively. These sequences appeared to be nearly identical to the apxIICA (clyIICA) genes of serotype 9 (Smits et al., 1991). Using PCR amplification, gene expression and monoclonal and polyclonal antibodies, the AppA protein was shown to be identical to ApxIIA and to be produced by all serotypes except 10 (Frey et al., 1992; Kamp et al., 1991; Jansen et al., $1992 a$; Smits et al., 1991). apxIIA is present in all serotype reference strains except 10 and shows no significant allelic variations (Frey et al., 1993; Jansen et al., 1992a). The apxII operon was shown to contain only the activator gene apxIIC and the gene for the structural protein apxIIA, but not the genes for the secretion proteins B and D (Jansen et al., 1992a; Frey et al., 1993; Chang et al., 1989; Smits et al., 1991) (Table 2). The secretion genes were probably deleted, since only short segments of B-like genes were found downstream of apxIIA (Chang et al., 1989; Smits et al., 1991). Secretion of the ApxIIA protein seems to occur by transcomplementation with $a p x I B$ and $a p x I D$ gene products (Frey et al., 1993; Jansen et al., 1992a). DNA sequence 
Table 2. Presence of the different apx genes in the reference strains for the different serotypes of $A$. pleuropneumoniae

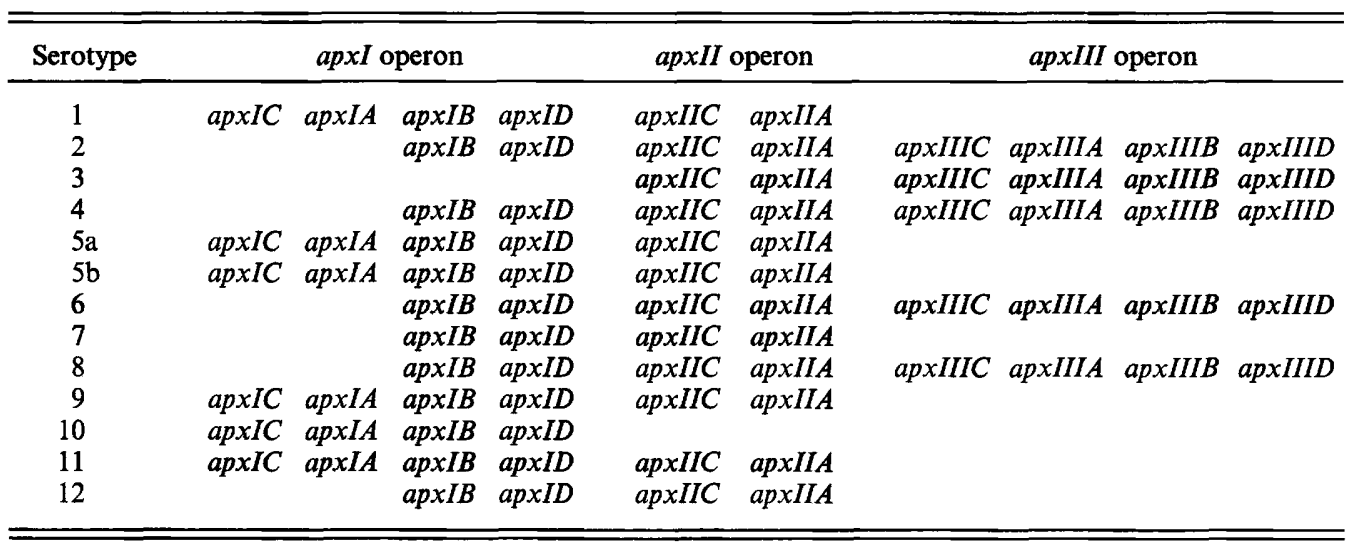

Data were compiled from: Jansen et al. (1992a,b); Frey et al. (1992, 1993); Gygi et al. (1992); Smits et al. (1991); Chang et al. (1989, 1991). See text for details.

analysis of apxIIA reveals that the ApxIIA protein has a calculated molecular mass of $102.5 \mathrm{kDa}$ and contains eight glycine-rich repeats (Chang et al., 1989; Smits et al., 1991). ApxIIA resembles the $P$. haemolytica leukotoxin LktA, and to a lesser extent ApxIA and the E. coli haemolysin HlyA (Smits et al., 1991; Chang et al., 1989). apxIIC and apxIIA were also cloned from serotype 7 and were named $c y t C$ and $c y t A$ respectively (Anderson et al., 1991; Rossi Campos et al., 1992) and were shown by DNA sequence analysis to be identical with the apxIICA sequences of serotype 5 and 9 (Chang et al., 1989; Smits et al., 1991).

\section{ApxIII (previously Ptx, ClyIII, Mat)}

The third RTX-toxin of $A$. pleuropneumoniae, ApxIII, is a protein with an apparent molecular mass of $120 \mathrm{kDa}$. ApxIII is present in the reference strains of serotypes 2, $3,4,6$ and 8 and was initially named cytolysin III (ClyIII) (Kamp et al., 1991; Jansen et al., 1992b, 1993), or pleurotoxin (Ptx) (Rycroft et al., 1991a; Macdonald \& Rycroft, 1992), or macrophage toxin (Mat) (van den Bosch et al., 1992; van den Bosch, 1991). ApxIII has no haemolytic activity but shows strong cytotoxic activity towards alveolar macrophages and neutrophils (Kamp et al., 1991; Rycroft et al., 1991 a; Macdonald \& Rycroft, 1992; van den Bosch, 1991). The gene apxIIIA for the structural protein ApxIIIA was cloned and expressed in $E$. coli and designated ptxA, or clyIIIA (Macdonald \& Rycroft, 1992; Jansen et al., 1992b). It was found to be present in the reference strains of serotypes $2,3,4,6$ and 8 (Macdonald \& Rycroft, 1992; Jansen et al., 1992b) (Table 2). In all these serotypes a complete operon consisting of the activator gene apxIIIC, the structural gene for the toxin apxIIIA, and two secretion genes
apxIIIB and apxIIID were found (Table 2) and previously named clyIIIC, clyIIIA or ptxA, clyIIIB and clyIIID respectively (Jansen et al., 1992b; Macdonald \& Rycroft, 1992). The DNA sequence of apxIIIC and apxIIIA from the serotype 8 reference strain has been established (Jansen et al., 1993). The derived amino acid sequence for ApxIIIA shows the typical features of RTX-toxins, with the three hydrophobic domains in the $\mathrm{N}$-terminal half of the protein and 13 glycine-rich repeats in the C-terminal part of the protein (Jansen et al., 1993). The calculated molecular mass of ApxIIIA is $112.8 \mathrm{kDa}$ (Jansen et al., 1993).

\section{Concluding remarks}

Detailed genetic and biochemical data are now available on $A$. pleuropneumoniae RTX-toxins. Their exact functions and target cells still remain to be established. It is therefore most important that all research groups working on these toxins use the uniform designations ApxI, ApxII and ApxIII proposed here, in order to avoid confusion and to ensure rapid research progress in the field.

We are grateful to Rodney Welch, Department of Medical Microbiology, University of Wisconsin, Madison, WI, USA, and to Colin Hughes, Department of Pathology, Cambridge University, Cambridge, UK, for valuable help and suggestions for the new toxin designations and to the organizers of the Fallen Leaf Conference on 'Bacterial Virulence Factors', South Lake Tahoe, CA, USA, for providing excellent opportunities for discussions.

\section{References}

Anderson, C., Potter, A. A. \& Gerlach, G. F. (1991). Isolation and molecular characterization of spontaneously occurring cytolysin- 
negative mutants of Actinobacillus pleuropneumoniae serotype 7 . Infection and Immunity 59, $4110-4116$.

Bhatia, B., Mittal, K. R. \& Frey, J. (1991). Factors involved in immunity against Actinobacillus pleuropneumoniae in mice. Veterinary Microbiology 29, 147-158.

VAN DEN BosCH, J. F. (1991). Actinobacillus pleuropneumoniae subunit vaccine. Patent application EP-0453024.

van den Bosch, J. F., Jongenelen, I. M. C. A., Pubben, A. N. B., van Vugt, F. G. A. \& Segers, R. P. A. M. (1992). Protection induced by a trivalent $A$. pleuropneumoniae subunit vaccine. Proceedings $12 t h$ IPVS, The Hague, The Netherlands, p. 194

Bosse, J. T., Johnson, R. P., Nemec, M. \& Rosendal, S. (1992). Protective local and systemic antibody responses of swine exposed to an aerosol of Actinobacillus pleuropneumoniae serotype 1. Infection and Immunity 60, 479-484.

BURROWs, L. L. \& Lo, R. Y. (1992). Molecular characterization of an RTX determinant from Actinobacillus suis. Infection and Immunity 60, 2166-2173.

Chang, Y. F., Young, R. \& Struck, D. K. (1989). Cloning and characterization of a hemolysin gene from Actinobacillus (Haemophilus) pleuropneumoniae. DNA 8, 635-647.

Chang, Y. F., Young, R. Y. \& Struck, D. K. (1991). The Actinobacillus pleuropneumoniae hemolysin determinant: unlinked $a p p C A$ and $a p p B D$ loci flanked by pseudogenes. Journal of Bacteriology 173, 5151-5158.

DEVENISH, J. \& Rosendal, S. (1991). Calcium binds to and is required for biological activity of the 104-kilodalton hemolysin produced by Actinobacillus pleuropneumoniae serotype 1. Canadian Journal of Microbiology 37, 317-321.

DeVEnish, J., Rosendal, S. \& Bosse, J. T. (1990a). Humoral antibody response and protective immunity in swine following immunization with the 104-kilodalton hemolysin of Actinobacillus pleuropneumoniae. Infection and Immunity 58, 3829-3832.

Devenish, J., Rosendal, S., Bosse, J. T., Wilkie, B. N. \& Johnson, R. $(1990 \mathrm{~b})$. Prevalence of seroreactors to the 104-kilodalton hemolysin of Actinobacillus pleuropneumoniae in swine herds. Journal of Clinical Microbiology 28, 789-791.

Devenish, J., Brown, J. E. \& Rosendal, S. (1992). Association of the RTX proteins of Actinobacillus pleuropneumoniae with hemolytic, CAMP, and neutrophil-cytotoxic activities. Infection and Immunity 60, 2139-2142.

Dom, P., Haesebrouck, F., Kamp, E. M. \& Smits, M. A. (1992) Influence of Actinobacillus pleuropneumoniae serotype-2 and its cytolysins on porcine neutrophil chemiluminescence. Infection and Immunity 60, 4328-4334.

Fedorka Cray, P. J., Huether, M. J., Stine, D. L. \& Anderson, G. A. (1990). Efficacy of a cell extract from Actinobacillus (Haemophilus) pleuropneumoniae serotype 1 against disease in swine. Infection and Immunity 58, 358-365.

Felmlee, T., Pellett, S., Lee, E. Y. \& Welch, R. A. (1985). Escherichia coli hemolysin is released extracellularly without cleavage of a signal peptide. Journal of Bacteriology 163, 88-93.

FREY, J. \& NiCOLET, J. (1988a). Recognition of hemolysin expression in Actinobacillus pleuropneumoniae serotype 1 by $\mathrm{Ca}^{2+}$. Infection and Immunity 56, 2570-2575.

FreY, J. \& NiCOLET, J. $(1988 b)$. Purification and partial characterization of a hemolysin produced by Actinobacillus pleuropneumoniae type strain 4074. FEMS Microbiology Letters 55, 41-46.

FREY, J. \& NiCOLET, J. (1990). Hemolysin patterns of Actinobacillus pleuropneumoniae. Journal of Clinical Microbiology 28, 232-236.

FreY, J. \& Nicolet, J. (1991). Immunological properties of Actinobacillus pleuropneumoniae hemolysin I. Veterinary Microbiology 28, 61-73.

Frey, J., Deillon, J. B., Gygi, D. \& Nicolet, J. (1991a). Identification and partial characterization of the hemolysin (HlyII) of Actinobacillus pleuropneumoniae serotype 2. Veterinary Microbiology 28, 303-312.

Frey, J., Meier, R., Gygi, D. \& Nicolet, J. (1991 b). Nucleotide sequence of the hemolysin I gene from Actinobacillus pleuropneumoniae. Infection and Immunity 59, 3026-3032.

Frey, J., van den Bosch, H., Segers, R. \& Nicolet, J. (1992). Identification of a second hemolysin (HlyII) in Actinobacillus pleuropneumoniae serotype 1 and expression of the gene in Escherichia coli. Infection and Immunity 60, 1671-1676.

FreY, J., BeCK, M., STUCKI, U. \& Nicolet, J. (1993). Analysis of hemolysin operons in Actinobacillus pleuropneumoniae. Gene 123, 51-58.

Gygi, D., Nicolet, J., Frey, J., Cross, M., Koronakis, V. \& Hughes, C. (1990). Isolation of the Actinobacillus pleuropneumoniae haemolysin gene and the activation and secretion of the prohaemolysin by the HlyC, HlyB and HlyD proteins of Escherichia coli. Molecular Microbiology 4, 123-128.

Gygi, D., Nicolet, J., Hughes, C. \& Frey, J. (1992). Functional analysis of the $\mathrm{Ca}^{2+}$-regulated hemolysin I operon of Actinobacillus pleuropneumoniae serotype 1 . Infection and Immunity 60, 3059-3064.

InZANA, T. J., TODD, J., MA, J. N. \& VeIT, H. (1991). Characterization of a non-hemolytic mutant of Actinobacillus pleuropneumoniae serotype 5: role of the 110 kilodalton hemolysin in virulence and immunoprotection. Microbial Pathogenesis 10, 281-296.

Issartel, J. P., Koronakis, V. \& Hughes, C. (1991). Activation of Escherichia coli prohaemolysin to the mature toxin by acyl carrier protein-dependent fatty acylation. Nature, London 351, 759-761.

Jansen, R., Briaire, J., Kamp, E. M. \& Smits, M. A. (1992a). The cytolysin genes of Actinobacillus pleuropneumoniae. Proceedings 12 th IPVS, The Hague, The Netherlands, p. 197.

JANSEN, R., Briatre, J., KAMP, E. M. \& SMits, M. A. (1992b). Comparison of the cytolysin II genetic determinants of Actinobacillus pleuropneumoniae serotypes. Infection and Immunity 60, 630-636.

Jansen, R., Briaire, J., Kamp, E. M., Gielkens, A. L. J. \& Smits, M. A. (1993). Cloning and characterization of the Actinobacillus pleuropneumoniae-RTX-toxin III (ApxIII) gene. Infection and Immunity 61, 947-954.

KAMP, E. M. \& VAN LeENGOED, L. A. (1989). Serotype-related differences in production and type of heat-labile hemolysin and heatlabile cytotoxin of Actinobacillus (Haemophilus) pleuropneumoniae. Journal of Clinical Microbiology 27, 1187-1191.

Kamp, E. M., Popma, J. K., Anakotta, J. \& Smits, M. A. (1991). Identification of hemolytic and cytotoxic proteins of Actinobacillus pleuropneumoniae by use of monoclonal antibodies. Infection and Immunity 59, 3079-3085.

Lalonde, G., McDonald, T. V., Gardner, P. \& O'Hanley, P. D. (1989). Identification of a hemolysin from Actinobacillus pleuropneumoniae and characterization of its channel properties in planar phospholipid bilayers. Journal of Biological Chemistry 264, 13559-13564.

VAN LeENGoed, L. A. \& Dickerson, H. W. (1992). Influence of calcium on secretion and activity of the cytolysins of Actinobacillus pleuropneumoniae. Infection and Immunity 60, 353-359.

MA, J. N. \& INZANA, T. J. (1990). Indirect enzyme-linked immunosorbent assay for detection of antibody to a 110000 molecular-weight hemolysin of Actinobacillus pleuropneumoniae. Journal of Clinical Microbiology 28, 1356-1361.

MaCDONALD, J. \& RYCroft, A. N. (1992). Molecular cloning and expression of ptxA, the gene encoding the 120-kilodalton cytotoxin of Actinobacillus pleuropneumoniae serotype 2. Infection and Immunity 60, 2726-2732.

Nicolet, J. (1992). Actinobacillus pleuropneumoniae. In Diseases of Swine, 7th edn, pp. 401-408. Edited by A. D. Leman, B. E. Straw, W. L. Mengeling, S. D'Allaire \& D. J. Taylor. Ames, Iowa: Iowa State University Press.

Rosendal, S., Devenish, J., Macinnes, J. I., Lumsden, J. H., Watson, S. \& XuN, H. (1988). Evaluation of heat-sensitive, neutrophil-toxic, and hemolytic activity of Haemophilus (Actinobacillus) pleuropneumoniae. American Journal of Veterinary Research 49, 1053-1058.

Rossi Campos, A., Anderson, C., Gerlach, G. F., Klashinsky, S., PotTer, A. A. \& Willson, P. J. (1992). Immunization of pigs against Actinobacillus pleuropneumoniae with two recombinant protein preparations. Vaccine 10, 512-518.

Rycroft, A. N., Williams, D., Cullen, J. M. \& Macdonald, J. (1991 a). The cytotoxin of Actinobacillus pleuropneumoniae (pleurotoxin) is distinct from the haemolysin and is associated with a $120 \mathrm{kDa}$ polypeptide. Journal of General Microbiology 137, 561-568.

Rycroft, A. N., Williams, D., McCandlish, I. A. \& TaYloR, D. J. $(1991 \mathrm{~b})$. Experimental reproduction of acute lesions of porcine 
pleuropneumonia with a haemolysin-deficient mutant of Actinobacillus pleuropneumoniae. Veterinary Record 129, 441-443.

SHOPE, R. E. (1964). Porcine contagious pleuropneumonia. Experimental transmission, etiology and pathology. Journal of Experimental Medicine 119, 357-368.

Smits, M. A., Briaire, J., Jansen, R., Smith, H. E., Kamp, E. M. \& GielKeNs, A. L. (1991). Cytolysins of Actinobacillus pleuropneumoniae serotype 9. Infection and Immunity 59, 4497-4504.
Stine, D. L., Huether, M. J., Moxley, R. A. \& SRIKumaran, S. (1991). Actinobacillus pleuropneumoniae-induced thymic lesions in mice and pigs. Infection and Immunity 59, 2885-2891.

UdEZE, F. A. \& KADIS, S. (1992). Effects of Actinobacillus pleuropneumoniae hemolysin on porcine neutrophil function. Infection and Immunity 60, 1558-1567.

WELCH, R. A. (1991). Pore-forming cytolysins of Gram-negative bacteria. Molecular Microbiology 5, 521-528. 